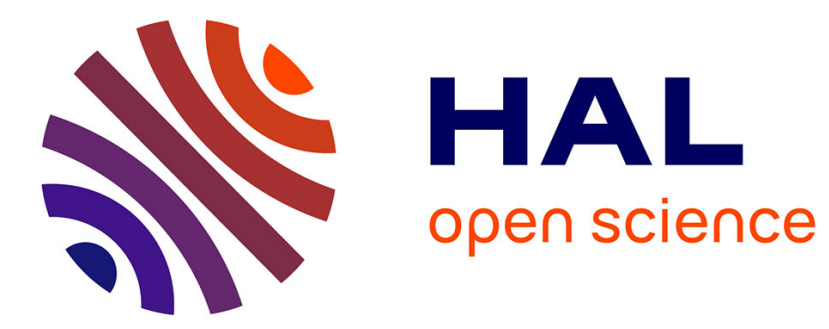

\title{
Figures et points de vue en confrontation
}

\author{
Alain Rabatel
}

\section{To cite this version:}

Alain Rabatel. Figures et points de vue en confrontation. Langue française, 2008, 4 (160), pp.3-19. 10.3917/lf.160.0003 . halshs-00367552

\section{HAL Id: halshs-00367552 https://shs.hal.science/halshs-00367552}

Submitted on 27 Mar 2018

HAL is a multi-disciplinary open access archive for the deposit and dissemination of scientific research documents, whether they are published or not. The documents may come from teaching and research institutions in France or abroad, or from public or private research centers.
L'archive ouverte pluridisciplinaire HAL, est destinée au dépôt et à la diffusion de documents scientifiques de niveau recherche, publiés ou non, émanant des établissements d'enseignement et de recherche français ou étrangers, des laboratoires publics ou privés. 


\title{
FIGURES ET POINTS DE VUE EN CONFRONTATION
}

\author{
Alain Rabatel
}

\section{Armand Colin I Langue française}

$2008 / 4-n^{\circ} 160$

pages 3 à 3

\section{ISSN 0023-8368}

Article disponible en ligne à l'adresse:

http://www.cairn.info/revue-langue-francaise-2008-4-page-3.htm

Pour citer cet article :

Rabatel Alain, «Figures et points de vue en confrontation »,

Langue française, $2008 / 4 \mathrm{n}^{\circ} 160$, p. 3-3. DOI : 10.3917/lf. 160.0003

Distribution électronique Cairn.info pour Armand Colin.

(c) Armand Colin. Tous droits réservés pour tous pays.

La reproduction ou représentation de cet article, notamment par photocopie, n'est autorisée que dans les limites des conditions générales d'utilisation du site ou, le cas échéant, des conditions générales de la licence souscrite par votre établissement. Toute autre reproduction ou représentation, en tout ou partie, sous quelque forme et de quelque manière que ce soit, est interdite sauf accord préalable et écrit de l'éditeur, en dehors des cas prévus par la législation en vigueur en France. II est précisé que son stockage dans une base de données est également interdit. 


\section{Figures et points de vue en confrontation}

Le présent dossier ne prétend pas apporter de cadre global à l'analyse des figures, ni n'ambitionne de procéder à une nouvelle typologie. En revanche, il traite des figures et des figures d'énonciateurs auxquelles elles concourent à partir de préoccupations énonciatives et pragmatiques qui ont fait l'objet à des degrés divers de plusieurs livraisons de Langue française, de Langages $^{1}$ et de bien $\mathrm{d}^{\prime}$ autres revues ou ouvrages avec lesquels ce travail est en consonance ${ }^{2}$. Il a semblé opportun aux auteurs de ce numéro, qui s'inscrivent dans le courant de l'analyse de discours, de développer à nouveau ces cadres d'analyse en accordant une place centrale à la notion ducrotienne de point de vue. Cette notion sera mise à contribution pour l'analyse des figures - qui plus est, de figures contextualisées - et non pas seulement des tropes, à l'instar de Forget 2000. Ce choix est stratégique: privilégier les tropes ${ }^{3}$ fait perdre de vue le caractère global du fait figural (Bonhomme 2005), de ses mécanismes et de sa fonctionnalité, en renforçant l'approche substitutive. Aussi ce dossier aborde-t-il des figures peu investiguées en linguistique (oxymore, antimétabole, tautologie,

1. Cf. Langue française 101 (Les figures de rhétorique, 1994), 113 (Aux sources de la polysémie nominale, 1997) 123 (Sémantique du stéréotype, 1999), 129 (Les figures entre langue et discours, 2001), 134 (Nouvelles approches de la métaphore, 2002), Langages 139 (La parole proverbiale, 2000), Cahiers de praxématique 35 (Sens figuré et figuration du monde, 2000), Semen 15 (Figures du discours et ambiguïté, 2001) etc.

2. Voir notamment Forget 2000, pour les figures de pensée et de discours, Détrie 2001, pour de très stimulantes analyses consacrées au déplacement des problématiques qui assouplissent le cadre structural de La rhétorique générale (Henry, Le Guern, Kleiber, Kerbrat-Orecchioni, Rastier, etc.), et Bonhomme 2005, pour le cadre général énonciatif et pragmatique. Le lecteur trouvera également dans les travaux de Landheer 2007 sur le paradoxe une analyse, basée sur le mécanisme de la dissimilation sémantique dans un cadre polyphonique, en congruence avec notre approche.

3. À la différence du $n^{\circ} 129$ de Langue française, qui compte un seul article sur une figure non trope, l'hypallage, trois articles sur la métaphore et un sur la métonymie. 
métalepse, énallage) ou tente-t-il d'enrichir la description de figures mieux connues (hyperbole, euphémisme, litote). Compte tenu du caractère inédit de l'analyse des figures à partir de la problématique énonciative du point de vue, l'introduction ne comportera pas un état de l'art et se limitera aux seuls aspects directement indispensables à la mise en œuvre de l'hypothèse de points de vue en confrontation. La première partie revient brièvement sur les débats déjà anciens concernant la définition des figures tropes et non tropes et les diverses conceptions de l'écart. La deuxième partie présente et exemplifie les notions d'énonciateur et de point de vue à l'œuvre dans la dynamique figurale. Enfin, la troisième partie esquisse les grandes lignes de la notion de confrontation de points de vue, dans le cadre du dialogisme interdiscursif et interlocutif, au cœur des articles de ce numéro.

\section{LES THÉORISATIONS DE L'ÉCART}

\section{I.I. Tropes et un seul mot et figures de pensée}

Bonhomme propose une définition de la figure compatible avec les conceptions restreignant la figure à une unité linguistique comme avec celles qui l'élargissent aux unités linguistiques dépassant les "tropes en un seul mot »: «Une figure est une forme discursive marquée, libre et mesurable, qui renforce le rendement des énoncés » (Bonhomme 1998 : 7). Cette compatibilité est précieuse, car, comme Perrin le souligne, la dichotomie entre trope en un seul mot et trope diffus est contestable :

Les tropes ne sont jamais autre chose que ce que les Anciens ont défini comme des figures de pensée. L'erreur fondamentale des rhétoriciens n'était pas de rapprocher l'ironie de la métaphore et d'en faire une figure de rhétorique, mais bien d'opposer les tropes dans leur ensemble aux figures de pensée. (Perrin $1996: 15)$

Si cette position est partagée par la plupart des tenants de l'analyse pragmatique des figures, et donc les auteurs de ce numéro, elle ne peut toutefois être défendue de façon radicale. En effet, même si l'on revendique une approche globale, et donc unitaire, des figures, on ne peut évacuer totalement la pertinence de l'opposition tropes vs figures de pensée. Car les tropes en un seul mot ont un foyer qui ne s'étend que sur un segment de la phrase et doublent l'incohérence avec le co(n)texte d'une incohérence interne. En revanche, dans les tropes diffus - cohérents d'un point de vue interne, à l'instar de l'allégorie ou des métaphores filées -, c'est tout un énoncé qui reçoit une interprétation figurée sur la base d'un conflit avec le cotexte (Prandi 2000). Une telle opposition ne garde cependant sa pertinence que si l'on ne survalorise pas la déviance linguistique au détriment de la déviance discursive : car un énoncé linguistiquement déviant ne l'est pas nécessairement sur le plan discursif (Nyckees 2000 : 119) - et inversement. C'est pourquoi les articles de ce dossier accordent de l'importance à la contextualisation des figures. 


\section{I.2. Les théories substitutives de l'écart}

Les figures sont le plus souvent analysées à partir des notions d'écart, de normes, qui servent à étalonner leur valeur. Ces notions sont au cœur des théories substitutives des figures, largement répandues. Il est vrai qu'il n'est pas facile de rendre compte de l'écart en échappant à la représentation d'une manière oblique de dire se substituant à une manière directe de s'exprimer - représentation qui, certes, n'est jamais formulée si directement par les linguistes, au fait de son caractère factice, mais qui a néanmoins pratiquement cours ${ }^{4}$.

Black traite de l'écart comme «jeu réciproque entre foyer et cadre » (Black 1978). Genette 1966 pense l'écart comme interaction entre texte/co(n)texte. Cohen 1966 analyse l'écart comme "impertinence prédicative », et le groupe $\mu$ en rend compte à partir d'un degré zéro défini comme « ce que le lecteur attend dans cette position » (Dubois et alii 1970 : 36). Dans un autre cadre théorique, les figures sont appréhendées comme activité cognitive (Lakoff et Johnson 1985), l'écart étant la trace d'un conflit entre concepts (Prandi 1992). Une autre approche repose sur la notion de "déviance » sémantico-pragmatique : dans la présentation du $\mathrm{n}^{\circ} 101$ de Langue française, Landheer l'évoquait comme «transgression de règles linguistiques (de sélection) », "parcours interprétatifs insolites » (Landheer 1994 : 6) et Kleiber, dans le même numéro, parlait, à propos de la métaphore, de «procédure de catégorisation non conventionnelle $»^{5}$.

Toutes ces analyses s'éloignent de la définition de l'écart comme «infraction » au code et remettent plus ou moins en cause l'opposition entre sens littéral et sens figuré, notamment en substituant aux propriétés internes inhérentes au contenu des expressions des propriétés applicables aux relations, en adéquation au contexte communicationnel ${ }^{6}$. Mais, centrées sur la réception, elles alimentent les théories substitutives, ne serait-ce que parce que le fait figural y est pensé en sous-estimant les calculs énonciatifs qui président au choix des modes de donation des référents du cotexte ${ }^{7}$. De même, les analyses pragmatiques de Grice, Searle, Kerbrat-Orecchioni 1986, 1994, alimentent le cadre substitutif en analysant les figures comme un écart entre un énoncé mani-

\footnotetext{
4. Ainsi les théorisations de la connotation présupposent toujours l'existence d'un discours purement dénotatif qui n'a d'autre réalité que théorique (Kerbrat-Orecchioni 1977/1984 : 222).
}

5. Détrie 2001 : 126s remarque que si la définition kleiberienne de la métaphore comme "acte de dénotation prédicative indirecte » est intéressante - parce qu'elle fait l'économie de la notion de connotation -, elle ne prend appui que sur le point de vue du récepteur : car du point de vue de l'émetteur, l'acte est direct.

6. «Une expression signifiante reçoit une interprétation littérale si le destinataire décide que le message intentionné ne contient rien de plus que le signifié de l'expression. Elle reçoit une interprétation non littérale si le destinataire décide que le message présente un contenu indépendant, qui doit être relié sur place au signifié de l'expression par une chaîne inférentielle motivée contextuellement. L'interprétation figurée n'est, de ce point de vue, "qu'un type d'expression non littérale aux propriétés spécifiques" » (Prandi 2000 : 21-22).

7. On trouve une tentative récente de replacer au centre de la figure les calculs énonciatifs et référentiels de l'émetteur dans Gaudin-Bordes \& Salvan (2008), pour l'hypallage et (à paraître) pour la paradiastole et l'antanaclase. 
festé déviant dont la pertinence n'apparaît qu'au terme d'un processus interprétatif se référant à des manières conventionnelles de dire et un énoncé littéral, en sorte que les figures s'analysent comme des tropes communicationnels (ou illocutoires) opposant un énoncé figural à un énoncé conventionnel qui respecterait les maximes conversationnelles.

La résistance des théories substitutives est étonnante alors que leurs limites sont connues et largement partagées : approche décontextualisée et paradigmatisante, survalorisation de quelques figures d'élocution (métaphore, métonymie, etc.), sous-évaluation des figures de pensée et d'invention, description réduite à l'énoncé sans prise en compte du discours, alimentation des approches dualistes de la langue et du style, puisque les figures n'affecteraient pas le fond de la pensée et se limiteraient à des tours de phrase qui ne visent que la "grâce ", indifférentes aux intentions pragma-énonciatives des locuteurs. À quoi on ajoutera que tous les écarts (fussent-ils affaiblis) ne sont pas des figures, $a$ fortiori des tropes, et que toutes les figures ne s'analysent pas comme écart.

Mais ces reproches visent fondamentalement moins l'écart que le cadre substitutif dans lequel on le pense. Si l'écart reste heuristiquement incontournable, il gagne à être traité en dehors des théories substitutives. D'où l'hypothèse selon laquelle l'écart renvoie

à une actualisation inattendue [d'un point de vue sur tel objet de discours] au regard d'une signification intersubjectivement stable, et dans l'appropriation toute personnelle de cette signification, qui la rend plus ou moins étrange, selon que la figure est plus ou moins conventionnelle. (Détrie 2000 : 9)

La saisie de l'écart opère ici dans le cadre d'une sémantique référentielle dans laquelle

les mots effectuent des découpages non du monde réel, mais du monde vécu par les sujets parlants, les mots reflétant, véhiculant avec eux des expériences multiples du sensible ${ }^{8}$, expériences anthropologiques, manipulatives, ou culturelles. (ibid.)

Une telle approche remet en cause les oppositions frontales sens littéral/sens figuré - mais pas les analyses qui pensent ces relations en terme de continuum, comme chez Culioli, Frankel et Lebaud. Elle interroge la théorie de la recomposition sémique (cf. la discussion autour de l'exemple de Jakobson, « la jeune fille est un bouleau »), qui mêle des significations en langue avec d'autres, qui relèvent davantage de l'extralinguistique (Molino, Soublin et Tamine 1979, Tamba 1981, Détrie 2001 : 71-72). Toutefois, de même que, en dépit de ses mérites, l'analyse syntaxique ne peut rendre compte de la spécificité des figures dans leur ensemble (Tamba 1981 : 188), la prise en compte des schèmes perceptifs ou des gestalt expérientielles ne suffit pas à rendre compte du fait figural dans sa totalité. Il n'est pas inutile de rappeler que ces limites concernent tous les cadres théoriques, y compris le cadre énonciatif que nous présentons ci-dessous.

8. Cf. les gestalts expérientielles chez Lakoff et Johnson. 


\section{LE CADRE ÉNONCIATIF DE L'ACTUALISATION DES POINTS DE VUE DANS LA DYNAMIQUE FIGURALE}

Nous nous appuierons sur la déliaison du locuteur/énonciateur primaire des énonciateurs intradiscursifs, afin de penser le fait figural comme une mise en scène énonciative de points de vue (PDV). Précisons, pour éviter tout contresens, que la notion de PDV à laquelle nous nous référons ici est celle de Ducrot 1984 : elle concerne l'ensemble des contenus propositionnels, indépendamment de leurs contenus sémantiques. Cette conception est donc plus large que celle que nous avions mise à contribution dans nos premiers travaux, consacrés aux perceptions, et à propos desquelles nous parlions de PDV représentés (Rabatel 1998 $)^{9}$. C'est sur la base de cet acquit ducrotien que nous avançons l'hypothèse de PDV en confrontation, afin de rendre compte des tensions intradiscursives au cœur de la dynamique figurale. Toutefois, l'exposé de cette hypothèse nécessite au préalable la clarification des relations entre locuteur, énonciateur et PDV, dans le cadre d'une conception de l'énonciation qui articule énonciation et référenciation (Benveniste 1974 : 82, Forest 1999, 2003, Rabatel 2005).

\section{I. Énonciateur et locuteur}

Benveniste conçoit l'énonciateur comme la source du mécanisme énonciatif en tant que tel, qu'il assimile aux seuls mécanismes d'embrayage : en ce sens, il n'y a pas d'énoncé sans énonciateur. $\mathrm{Si}$, en revanche, l'énonciateur est une position discursive permettant d'appréhender les objets du discours en lien ou non avec la situation d'énonciation, par centrage, décentrage, excentrage (Culioli) ou comme la source énonciative d'un PDV (Ducrot) distinct du locuteur/énonciateur premier (= L1/E1), l'énonciateur second (= e2) se définit comme l'instance dialogique de l'altérité énonciative, renvoyant à des PDV internes à l'énoncé par rapport auxquels L1/E1 se positionne.

Ces deux conceptions gagnent à être articulées. L'énonciateur E1 est donc l'énonciateur primaire, en syncrétisme avec le locuteur primaire ${ }^{10}$, celui qu'on a proposé de nommer principal - parce qu'il subsume tous les contenus propositionnels qu'il assume, parce que c'est à lui que les interlocuteurs comme les autorités demandent des comptes, le cas échéant (Rabatel 2005 : 126-127). L1/ E1 prend en charge ses propres PDV ${ }^{11}$. Quant aux PDV des énonciateurs seconds

9. Pour un examen global de ces différentes conceptions, nous nous permettons de renvoyer à Rabatel 2008a et b.

10. Le locuteur est l'instance qui profère un énoncé, dans ses dimensions phonétiques et phatiques ou scripturales, selon un repérage déictique ou selon un repérage indépendant d'ego, hic et nunc. La majuscule, suivie du chiffre 1, code le locuteur/énonciateur primaire, dont le rôle domine les locuteurs/énonciateurs seconds (12/e2). La barre oblique indique le syncrétisme de L1 et de E1 ou de 12 et de e2. On code e2 seul, en l'absence d'acte de parole : cf. infra, l'analyse de (1).

11. Tout énoncé présuppose une instance qui prend en charge ce qu'on appelle, selon les cadres de référence, dictum, lexie, contenu propositionnel, prédication, suivant la structure " JE DIS ("ce qui est $\operatorname{dit}^{\prime \prime}$ ». Au-delà des différences de dénomination, l'instance qui prend en charge un énoncé monologique est celle qui est à la source du processus de production de l'énoncé, en l'occurrence L1/E1. 
(ou internes), L1/E1 les leur impute et il les prend en compte, selon des positionnements qui peuvent se limiter à une prise en compte neutre, mais qui peuvent aussi aller jusqu'à l'expression d'un accord ou d'un désaccord plus ou moins nets, en fonction des marques d'approbation ou de distanciation. Autrement dit, dans un énoncé monologique, le locuteur est aussi énonciateur ; dans un énoncé dialogique, peuvent coexister un certain nombre de locuteurs/énonciateurs seconds - en un nombre théoriquement infini mais pratiquement limité par la gestion de la récursivité (Philippe 2005) -, limite accrue lorsque les énonciateurs renvoient à des PDV qui ne relèvent pas d'un acte de parole explicite proféré par un locuteur second (Ducrot 1984 : 204-205 et infra).

La distinction des énonciateurs primaires et seconds évite de réduire l'énonciation, comme fait Benveniste, aux discours en je ancrés dans la situation d'énonciation (Ono 2007) et de cantonner la problématique du PDV à la subjectivité du je: après tout, un PDV n'a pas besoin d'être personnel pour exister en tant que tel, et, de même, ce n'est pas parce qu'un contenu propositionnel ne comporte pas de subjectivèmes qu'il n'exprimerait pas un PDV (Rabatel 2005 : 117-120). La disjonction locuteur/énonciateur permet aussi d'envisager que les subjectivèmes ne soient pas tous rapportés à L1/E1, mais aussi à des énonciateurs seconds à partir desquels les objets du discours sont référés. C'est en ce sens que Ducrot 1989 traçait un parallèle avec le sujet modal de Bally : de fait, les énonciateurs seconds sont comparables à des centres de perspective, dont on peut reconstruire le PDV, y compris en l'absence de tout acte de locution, à travers le mode de donation des référents. Ces phénomènes, bien analysés à propos du discours représenté - avec diverses modalités d'absorption du PDV de l'autre dans la parole du locuteur citant, dans le discours indirect libre, le discours narrativisé, le PDV représenté, les îlots textuels, la modalisation autonymique - concernent aussi les figures de pensée et les tropes.

Examinons brièvement (1) à la lumière de ce cadre théorique :

(1) Les éléphants, les jeunes lions et la gazelle (Titre) ${ }^{12}$

La jungle du PS vient de s'enrichir de nouveaux animaux. Après les éléphants (les chefs de courant comme Laurent Fabius ou Dominique StraussKahn) et la gazelle (Ségolène Royal) les «jeunes lions » débarquent. Dans l'euphorie de sa victoire, Arnaud Montebourg a proclamé l'arrivée de ces félins destinés à remplacer les vieux pachydermes, qu'ils jugent usés. Le problème, c'est que le résultat du second tour des élections législatives atténue fortement les coups de griffe qu'ils pourraient vouloir donner. (Le Point, 21-6-2007)

En (1), le journaliste (L1/E1) évoque sur un mode distancié une jungle socialiste bien fournie, ainsi que l'indiquent les modalisations de la dernière phrase, qui confirment la distanciation ironique sensible dans le verbe "débarquer » et dans la mention de "l'euphorie de la victoire», circonstance censée expliquer l'imprudence de la rodomontade. Il n'en reste pas moins que les «jeunes

12. Les exemples ont été relevés lors de la campagne des élections présidentielles de 2007 en France, afin d'illustrer la fonction pragmatique des figures. On en trouvera d'autres dans l'article consacré aux antimétaboles. 
lions », en tant que métaphore, jouent un rôle de sujet modal, puisque la référenciation du discours est organisée à partir de leur PDV, quand bien même ils n'ont rien dit: la preuve en est que l'accord du verbe «jugent» se fait non sur Montebourg, comme on aurait pu s'y attendre, mais sur les félins eux-mêmes, qui trouvent les pachydermes "usés ». Dès lors, tout ce qui précède, sans annuler la distanciation ironique de L1/E1, peut se lire en surimpression comme une déclaration de guerre : « attention, les éléphants, nous débarquons ! ». Comme le rappelle Ducrot 1984: 204, de tels énonciateurs existent dans le discours sans être locuteurs :

J'appelle 'énonciateurs' ces êtres qui sont censés s'exprimer à travers l'énonciation, sans que pour autant on leur attribue des mots précis ; s'ils 'parlent', c'est seulement en ce sens que l'énonciation est vue comme exprimant leur point de vue, leur position, leur attitude, mais non pas, au sens matériel du terme, leurs paroles. (Ducrot 1984 : 204)

L'énonciateur second existe dans le discours de L1/E1 sous la forme de traces, dans le dictum. C'est pourquoi le rapprochement avec le sujet modal esquissé par Ducrot 1989 ne doit pas faire conclure que e2 ne serait présent que dans le modus : e2 est partout, dans les choix de dénomination, de qualification et de structuration du dictum (Ducrot 1993).

\subsection{L'empan du point de vue}

On peut fonder l'empan du PDV sur la notion de prédication, moyennant quoi le PDV ne doit pas être inférieur à un énoncé complet (énoncé ou phrase simple) : la prédication est le cadre de l'assertion prise en charge par le locuteur. Cette conception est particulièrement pertinente pour les locuteurs/ énonciateurs primaires. Mais si l'on considère la langue dans sa dimension dialogale, dialogique et interactionnelle, le PDV peut se limiter à la reprise d'un mot ou d'un groupe de mots, à la manière des îlots textuels ou des modalisations autonymiques. Dans ce cas, il n'y a pas de limite inférieure au PDV, ce dernier, embryonnaire, existe en langue, en fonction de l'orientation argumentative des lexèmes (Carel et Ducrot 1999, Schulz 2001). Il se manifeste également par accrétion dans le discours, à travers le choix d'un terme suffisamment emblématique dans une société pour renvoyer à une position, à une vision du monde ou à une manière de dire particulières. Ces mots-étendards porteurs de PDV ont des durées de vie variable, selon les contraintes des communautés discursives : ainsi de "racaille », "nettoyer au karcher », «jurys populaires » durant la dernière campagne de l'élection présidentielle.

Inversement, à l'échelle du texte ou de vastes corpus, il paraît légitime de rassembler en un PDV global les PDV relevant d'une même isotopie, de la même source ou de la même orientation argumentative (même si les sources varient), dès lors qu'ils convergent dans l'expression d'une vision du monde partagée. Bref, l'empan du PDV est plus ou moins large, suivant les éléments pris en compte. Cette question est importante pour l'analyse des figures, le PDV qu'elles construisent pouvant aller du mot ou du groupe de mots (« jeunes 
lions ", en (2)) à la prédication d'une assertion en (3), voire se déployer sur un texte, comme le fait la métaphore filée de (2) :

(2) Il y a eu le temps des éléphants, désormais révolu, et dont la page se tourne définitivement. Voici maintenant venu le temps enthousiasmant des jeunes lions : sereins, puissants et capables des meilleurs combats. (A. Montebourg, 17-6-2007)

(3) Un éléphant ne craint pas le lion. (H. Emmanuelli, Le Monde 20-6-2007)

(2), prononcé à l'issue victorieuse d'un combat difficile, alourdi par les conflits internes au parti socialiste, met en scène un député qui, en tant que locuteur/énonciateur principal, annonce un changement d'époque, marqué par l'antithèse des tiroirs temporels opposant le passé composé il y a eu, le participe passé révolu au présent actuel du commentaire dont la page se tourne définitivement. L'ère nouvelle, évoquée par un participe en lien avec des marqueurs déictiques (voici maintenant venu), repose sur un chiasme $(\mathrm{N}+$ groupe adjectival vs adjectif $+\mathrm{N}$ ), puis se renforce par l'inclusion de métaphores filées sur l'ensemble de ce micro-texte. Les éléphants comme les lions sont délocutés. Mais le contraste est fort entre les éléphants que L1/E1 évoque de l'extérieur en signalant leur disparition, et les lions affublés de qualificatifs positifs et dotés d'un programme narratif dynamique. La prégnance des subjectivèmes positifs invite à considérer les lions comme un sujet modal, et en surimpression, on entend le PDV de celui qui dit nous/je: nous, les lions, sommes jeunes, sereins/je suis particulièrement capable des meilleurs combats.

Le processus figural fait corps avec l'ensemble du mode de donation des référents des énoncés. Son analyse, en tant que PDV, est intéressante à plusieurs titres :

- Elle inscrit la figure dans un co-texte dont certes elle est le joyau, tout en rappelant (filons la métaphore) qu'il n'est pas de joyau sans écrin...

- Elle articule dimension expressive et intention significative/communicative, en invitant à prendre en compte sa dimension pragmatique, quand bien même, comme on va le voir, la figure connue et reconnue est décontextualisée pour être réensemencée dans un nouveau contexte.

- Elle repose sur des processus dialogiques actifs en production et en réception, en langue et en discours.

- Elle souligne que la dynamique figurale repose sur des PDV d'empan variable. Tandis que les éléphants sont posés pour souligner leur disparition dans le cours politiques des choses, les lions sont "actualisés », en tant que sujet modal, puisque l'univers de discours est organisé à partir d'eux. Les lions n'en restent pas moins porteurs d'un PDV embryonnaire négatif : la mention des "éléphants », en politique (et uniquement à propos du parti socialiste), porte en elle par accrétion un ensemble de représentations négatives. Le discours de L1/E1 joue avec ces PDV plus ou moins développés (ce qui en soi est un signe de leur importance) qui construisent une dynamique figurale complexe. Cette dynamique joue également à propos de (3), surtout si on le restitue dans son cotexte :

(4) Chacun sait qu'un éléphant ne craint pas le lion. 
L1/E1, qui ne partage pas certains choix d'A. Montebourg, conteste la pertinence de (2) en filant/retournant la métaphore filée animalière tout en s'appuyant sur un PDV doxique (dans le dictum). Il renforce son PDV dans le modus par un «chacun sait» qui en appelle à la complicité de l'opinion... On pourrait analyser (4) comme la mention par L1/E1 d'un seul PDV doxique dont il partage le propos, mais il semble plus juste de l'analyser comme la somme de deux énonciateurs et de deux PDV doxiques différents : l'énonciateur du dictum est un énonciateur doxique générique, le présent a une valeur intemporelle, l'assertion énonce une vérité générale. L'énonciateur indéterminé du modus est inscrit dans l'aire spatio-temporelle où interagit L1/E1 : chacun sait, en général et surtout parmi nous, ici et maintenant: le modus se charge d'une modalité déontique implicite (chacun devrait savoir) qui s'accompagne d'une mise en garde politique et d'une menace à peine voilée.

\subsection{La dimension pragmatique des PDV dans les figures}

L'analyse pragmatique des figures participe à la construction de l'ethos, de l'idiolecte des locuteurs (Détrie et Neveu 2005). Ainsi, les réticences, euphémismes peuvent contribuer à l'image d'un L1/E1 peu sûr de lui, hésitant, ménageant sa position ou la face de l'autre. L'hyperbole peut être interprétée comme une flatterie, l'ironie ou l'antithèse comme une affirmation de soi au détriment des autres. De telles motivations sociales et psychologiques sont infinies et toujours en lien avec le co(n)texte.

Les figures jouent un rôle privilégié dans la construction de figures d'énonciateurs spécifiques, en raison de leurs mécanismes de saillance et de prégnance. Mais ce rôle ne joue que dans un co-texte signifiant de part en part, et par rapport au contexte, ce qui nécessite une saisie articulée du texte et des interactions, comme l'a esquissé l'analyse de (2) et de (4). C'est dans ce cadre que l'étude pragmatique des interactions entre énonciateurs dans la co-construction des PDV s'ouvre sur la problématique de la surénonciation, de la sousénonciation ou de la coénonciation ${ }^{13}$. En (4), L1/E1 fait preuve d'une perspective surplombante de surénonciation en reprenant la métaphore filée tout en la retournant contre son auteur, en s'appuyant sur la complicité elle-même en surplomb avec les destinataires additionnels du message ${ }^{14}$. En (5), la sousénonciation apparaît dans la métaphore filée ${ }^{15}$ par laquelle un député communiste évoque le succès inespéré de son parti en inscrivant les limites de son succès dans le cadre de la reconnaissance d'une victoire de l'autre camp, qui reste la donnée structurante du discours :

13. La place nous manque pour développer cette question : voir Langages 156.

14. Cf. " Alors quand tout à son euphorie Montebourg déclare, "il y a eu le temps des éléphants désormais révolu, voici maintenant le temps enthousiasment [sic] des jeunes lions" sans doute oubliet-il que les éléphants survivants ont une mémoire d'éléphant!» http://www.reponseatout.com/ blog/index.php/2007/06/21/297-un-jeune-lion-a-lassaut.

15. Le choix d'une même figure montre que les figures ne relèvent pas par nature d'une seule posture énonciative. 
Eh bien c'est peut-être la vague bleue, mais dedans il y a un petit poisson rouge. (Lecoq, Antenne 2, 18-6-2007)

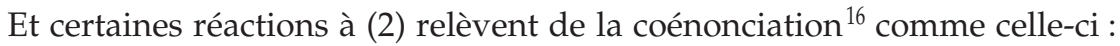

(6) OUAIS! Montebourg a déjà dit hier que le temps des éléphants étaient révolus [sic] et qu'il fallait laisser les jeunes refonder le PS. Montebourg 1-les éléphants 0

(http:/ / forumsocialiste.free.fr/viewtopic.php?t=11729\&sid=f6e82f328d97094 bfa521d399b8b70da)

L'analyse des figures fait sens dans des co(n)textes profondément dialogiques. Toutefois, le dialogisme rassemble un nombre de phénomènes si variés qu'il est indispensable de réduire l'analyse à des phénomènes signifiants dans un cotexte étroit : c'est dans ce cadre que la dynamique énonciative est travaillée par la notion de PDV en confrontation.

\section{LE DIALOGISME DE LA DYNAMIQUE FIGURALE ET LA CONFRONTATION DES POINTS DE VUE}

\section{I. L'actualisation des figures}

La figure exhibe le fait que les mots ne produisent pas du sens en tant que miroir des choses du monde extralinguistique ; ils construisent une représentation linguistique d'un réel extralinguistique par une appropriation personnelle du monde sensible (Détrie 2000 : 3), dans laquelle, comme le dit Authier-Revuz, «les mots ne vont pas de soi », et reposent sur de multiples non-coïncidences des mots à eux-mêmes et des mots aux choses. La formulation comme l'interprétation des figures reposent sur une "construction interactive du sens, comme un phénomène énonciatif qui rejoue les découpages stéréotypés » (Détrie 2000 : 10). C'est par rapport à ces données qu'intervient la notion de PDV en confrontation.

De quoi s'agit-il? Le processus figural repose sur l'actualisation inattendue d'un PDV au regard des manières habituelles de penser et de dire, en appui sur la singularité d'une expérience sensible et de son vouloir dire. Les PDV en confrontation sont en tension entre une dimension interactionnelle (confrontation agonique de PDV) et une dimension cognitive. La prédication n'est pas obligatoirement conflictuelle, elle peut projeter deux domaines

16. La coénonciation, comme co-construction d'un PDV commun partagé, diffère de la coénonciation culiolienne dans laquelle le locuteur construit son discours en fonction de l'auditeur (ou de l'auditoire) et de ses réactions. Elle est aussi distincte de la coénonciation comme co-construction de la signification du fait figural par le récepteur solidaire de l'échange : «la responsabilité de la production du sens rhétorique est répartie sur les deux partenaires, chacun en assumant une partie distincte» (Klinkenberg 2000 : 72). Dans ce dernier cas, des manipulations contextuelles destinées à sauvegarder le principe de coopération obligent le récepteur 1) à ne pas se satisfaire d'un ou plusieurs éléments à la surface de l'énoncé (= « degré perçu ») 2) à produire un ensemble d'interprétations qui se superpose - et non se substitue - au degré perçu, en un ensemble flou d'interprétations nommé « degré conçu » (ibid. : 64-65). 
notionnels ou deux espaces mentaux (Fauconnier 1984) l'un sur l'autre, à l'instar des travaux de Black 1962 et 1978, Cadiot 2001 sur la métaphore ou de ceux de Forget 2000 sur les figures de pensée. Autrement dit, confronter des PDV, ce n'est pas seulement les opposer violemment pour n'en retenir qu'un, $c^{\prime}$ est éventuellement les cumuler, voire les mettre en perspective, sur un mode explicite ou allusif, etc.

Ces PDV sont des lieux privilégiés d'une énonciation problématisante, opacifiant le dit/dire, volontairement ou non, dans la mesure où les mises en rapport soulèvent de nouvelles questions. Ils se manifestent de diverses manières : en s'opposant au contexte (ironie, litote), en opposant dans le cotexte des PDV différents co-présents (oxymore, comparaison, énallage, hypotypose, hyperbate, chiasme). Dans d'autres cas, l'opposition de PDV opère in absentia par rapport à une manière conventionnelle de dire (euphémisme, astéisme, enthymème). Ainsi, même lorsque la figure repose sur la réitération et la densification d'éléments appartenant à un ou plusieurs niveaux (phonétique, morphologique, syntaxique ou sémantique, cf. infra note 20), la notion de PDV en confrontation reste pertinente par rapport aux manières de dire moins marquées.

Sur un plan syntaxique, les choses sont complexes: les PDV en confrontation peuvent se manifester à l'intérieur d'une seule prédication (zeugme, oxymore), dans des prédications distinctes (antimétabole), voire irriguer un texte, comme en (1). On pourrait faire l'hypothèse que les tropes en un seul mot correspondent à des PDV embryonnaires (métaphores, oxymores), que certains tropes diffus ou figures de pensée relèvent davantage d'énonciateurs seconds organisant des prédications complètes (tautologies, antimétaboles, métaphores filées). Si cela se vérifie pour un trope comme l'oxymore, il est difficile de généraliser pour la métaphore, lorsqu'elle joue sur un double sens et tend vers la métaphore filée :

(7) Les électeurs du Modem se sont reportés à haut débit sur le PS. (Libération, 12-6-2007)

Dans le même ordre d'idée, une «même » figure, telle l'antithèse, peut se déployer à l'intérieur d'une seule prédication (8) ou sur des prédications différentes (9) :

(8) Monsieur Sarkozy est adepte des petites carottes et des gros bâtons. (B. Thibault, Libération, 1-3-2007)

(9) «Ce n'est pas parce que vous avez Judas à votre table qu'il faut vous prendre pour le Messie. (S. Royal, Le Monde 7-5-2007 ${ }^{17}$ )

\subsection{Un processus de production en tension entre singularité et régularité}

Selon Bonhomme,

17. Réplique que S. Royal avait prévu d'adresser à N. Sarkozy, lors du débat d'avant le second tour, s'il avait évoqué le ralliement d'E. Besson. 
Une figure constitue le plus souvent un compromis de singularité (qui la rend remarquable) et de régularité (qui la rend mesurable sur la chaîne des énoncés).

(Bonhomme 2005 : 70)

La tension entre singularité et régularité se concrétise aux plans morphosyntaxique, sémantique, référentiel, où se construisent des PDV en confrontation. Certes, la notion de PDV en confrontation fait immédiatement sens au plan sémantique, mais elle intervient en fait à tous les plans. Compte tenu des limites de ce dossier, nous n'abordons pas ici la problématique des PDV en confrontation aux plans morphologique ou phonétique, qui peuvent apparaître lors opérations de permutation, suppression, adjonction ${ }^{18}$. En revanche, nous privilégions certaines figures à base syntaxique qui reposent sur des opérations de déplacements, ruptures, amplifications, oppositions qui nous ont paru propices ${ }^{19}$ à l'examen des PDV en confrontation : c'est ainsi que les énallages et les métalepses (figures par rupture) sont analysées dans les articles de G. Salvan et de C. Détrie, que les tautologies (figures par amplification) font l'objet de l'article de L. Gaudin, que l'oxymore et les antimétaboles (figures par opposition) sont traités dans les articles de M. Monte et de A. Rabatel. Deux articles reviennent également sur des figures à base référentielle qui reposent sur des opérations de sur-sous-détermination, discordance: la problématique des PDV en confrontation y éclaire d'un jour nouveau les figures de la litote et de l'euphémisme, de l'hyperbole dans les articles d'A. Jaubert et de B. Verine.

Cette problématique traverse tous ces plans d'organisation du langage, portant soit sur la confrontation d'une occurrence avec le texte ou le contexte (Bonhomme parle de «figure par émergence singulière ») soit sur la réitération régulée de ces occurrences («figures par coémergence régulière ») ${ }^{20}$. Ces opérations et marques rendent compte de la saillance particulière des énoncés figuraux, au plan des signifiants; elles analysent l'écart par rapport aux fonctionnements ordinaires du langage et à l'intérieur de ces fonctionnements ordinaires, en appui sur un certain nombre de mécanismes de prégnance

18. Figures par permutation : anagramme, palindrome, verlan, contrepet. Figures par suppression : aphérèses, syncope, apocope, siglaison. Figures par adjonction : prosthèse, épenthèse, motvalise. Figures par déplacement: hypallage, chiasme, hyperbate. Certaines de ces figures feront l'objet d'une publication consacrée aux Figures de l'à-peu-près, à paraître au Français moderne.

19. Bien évidemment ces opérations jouent aussi pour les figures sémantiques.

20. Ces saillances jouent seules (émergence singulière) ou combinées (coémergence régulière) :

Figures par émergence singulière:

- par contraste cotextuel, correspondant à une saillance unipolaire: effacement (ellipse, syncope, apocope) ; inversion (palindrome, anagramme) ; décrochage (rupture d'isotopie, coq-àl'âne, syllepse); collision (polyphonie, message multicanal).

- Par contraste contextuel (euphémisme).

- Par contraste conceptuel (antonomase, euphémisme).

Figures par coémergence régulière, correspondant à une saillance bi- ou multipolaire :

- Par coémergence phonétique (homéothéleute, rime, contrepet) ;

- Par coémergence syntaxique constante (anaphore rhétorique, anadiplose, épiphore) ;

- Par coémergence syntaxique variable expansive (gradation), inversive (chiasme), oppositive (antithèse).

- Par coémergence textuelle (prosopopée) (Bonhomme 2005 : 58-67). 
psycholinguistiques ${ }^{21}$. Tel est le cadre général, profondément dialogique, à l'intérieur duquel seront analysés ces PDV en confrontation: il permet de penser dans la langue et à travers la dynamique discursive les rapports des locuteurs à la langue, aux interactants, aux référents, en se positionnant par rapport aux préconstruits linguistiques et aux cadres de pensée doxiques.

Certes, l'analyse globale du fait figural, étendue aux figures grammaticales (Verine 2000, Détrie et Verine 2003, Bres 2005), pourrait exposer la notion de figure au risque de la dilution. Mais la prise en compte des schèmes discursifs exemplaires et repérables sur la base de préconstruits linguistiques (Bonhomme 2005 : 29-33) offre un garde-fou utile pour une étude dialogique globale des tropes comme pour l'ensemble «des figures du discours autres que les tropes ${ }^{22}$ - parmi lesquelles Fontanier distingue l'interrogation, la concession, la correction, la délibération, la conglobation, le dialogisme, etc. Tel est le cadre à l'intérieur duquel se situent les auteurs de ce numéro.

\section{PRÉSENTATION DES ARTICLES}

Alain Rabatel traite des antimétaboles. Il analyse d'abord les relations entre antimétabole (répétition de mots dans un ordre inverse, dans des propositions successives), chiasme et réversion, avant de dégager deux grandes classes d'antimétabole, notées antimétabole PLUS $(\mathscr{A}+)$ et antimétabole MOINS $(\mathscr{A}-)$, selon que les points de vue (PDV) contenus dans les propositions inversées se conjuguent ou s'excluent. La saisie de ces PDV en confrontation dégage deux fonctionnements de l'antimétabole : dans $l^{\prime} \mathscr{A}+$, la hiérarchisation n'empêche pas le cumul des deux PDV pris en charge par L1/E1, tandis que dans $1^{\prime} \mathscr{A}-$, la hiérarchisation implique un rejet ou une distanciation du PDV pris en compte au profit du seul PDV pris en charge par L1/E1. Cette distinction rejaillit sur les diverses figures pragmatiques d'énonciateur qui émergent en discours, en tension entre la saisie du même et de l'autre, alimentant des figures d'énonciateur consensuelles et/ou agoniques.

21. La dynamique figurale repose notamment sur un processus de paradigmatisation - les figures exemplaires sont extraites de leur contexte énonciatif d'origine pour être intégrées dans des paradigmes plus abstraits - et sur un processus complexe d'exemplarisation qui s'appuie sur des mécanismes de prégnance psycho-linguistiques: les figures privilégient le préconstruit syntaxique et sémantique ("prégnance logico-conceptuelle») et les canevas rythmiques ("prégnance pulsionnelle») organisant le discours. Ces formes de prégnance se combinent avec la prégnance statistique, qui favorise la reconnaissance et la notoriété, ou avec la prégnance expériencielle, qui repose sur le principe selon lequel « plus une variation discursive traduit une expérience forte ou aisément vérifiable dans la vie courante, plus elle semble exemplaire et accède au statut de figure » (Bonhomme 2005 : 35-38).

22. Expression employée par Fontanier. Les Figures du discours, à la gloire de l'approche substitutive, tracent néanmoins des perspectives inédites autour du rapport entre les figures répertoriées par la rhétorique et les figures du discours autres que tropes, prolongeant ainsi les analyses (pré-)sémantiques des figures de Dumarsais (Douay-Soublin 1994). 
Michèle Monte propose de considérer l'oxymore comme la confrontation de points de vue contradictoires construits par le co(n)texte et rapportés à des sources énonciatives différentes. Elle met en lumière deux sortes d'oxymores, l'un à vocation polémique, l'autre à vocation de synthèse paradoxale. Dans le premier, le locuteur assume un seul des points de vue et présente le point de vue antithétique comme mensonger ou limité. Dans le second, le locuteur prend en charge un point de vue qui résulte de la somme des deux points de vue contradictoires. L'étude, appuyée sur des exemples littéraires contextualisés appartenant à des genres différents et sur des usages oraux contemporains, présente ensuite les enjeux textuels et pragmatiques de l'oxymore qui apparaît non seulement comme une arme argumentative contribuant à construire une posture de surénonciation mais aussi comme un outil de cohésion textuelle, propre à garder la mémoire de l'élaboration discursive en cours.

Lucile Gaudin réévalue le statut figural de la tautologie, dont la tradition rhétorique fait tantôt une figure "expressive » tantôt un vice, en mettant en avant son fonctionnement énonciatif et pragmatique à partir d'analyses contextuelles précises. La comparaison avec les figures approchantes du pléonasme et de l'antanaclase permet dans un premier temps de montrer que la dissimilation sémantique à l'œuvre dans la formule/X est $X /$, bien décrite par ailleurs, se double d'une assimilation forcée des points de vue en présence. L'étude souligne dans un deuxième temps le goût du tautologue pour l'expression objectivante qui lui permet d'afficher le seul point de vue doxique avant d'envisager dans un troisième temps les stratégies discursives auxquelles se prête la figure pour imposer à l'autre, via les postures énonciatives de coénonciation ou surénonciation, l'évidence d'une vision sans prise en charge manifeste.

Geneviève Salvan réexamine la métalepse dans une perspective pragmaénonciative, afin de rendre compte de l'hétérogénéité de ses définitions et de son empan variable - du trope temporel périmétonymique de $\mathrm{M}$. Bonhomme à la figure narratologique de G. Genette. Elle montre d'abord qu'aux critères consensuels de réordonnancement (chrono)logique et de désignation indirecte s'ajoute un dire décalé et une sélection de point de vue qui produisent un raccourci à la fois référentiel et énonciatif. Elle examine ensuite la nature de ce raccourci para-informatif et les parcours dynamiques des points de vue sur le même objet. L'étude de la mise en scène des points de vue et des stratégies discursives de l'énonciateur principal qui en découlent permet enfin de pointer les valeurs pragmatiques de la métalepse.

Catherine Détrie revisite l'énallage, qui signale l'emploi inattendu d'une forme grammaticale (tiroir verbal, mode ou personne) au regard du préconstruit grammatical et référentiel. Les explications proposées pour rendre compte de la distorsion perçue - substitution (Fontanier), phénomène d'ellipse syntaxique (Beauzée), voire faute (Dumarsais) - invitent à une "traduction »/ reconstruction de l'énoncé. Son analyse, consacrée à l'énallage de la personne, prend le contrepoint de ces approches en partant de la matérialité discursive (la tension des personnes inscrite dans la séquence figurale) pour décrire les positionnements énonciatifs sous-jacents, et les points de vue liés à ces positionne- 
ments. En inscrivant dans/par la configuration personnelle qui la caractérise la disjonction entre instances déictique et modale, l'énallage de la personne révèle simultanément la confrontation des points de vue et sa résolution.

Anna Jaubert procède à une analyse pragmatique de l'euphémisme et de la litote. Les figures dites de « style » sont aussi des manifestations d'actes de langage. Des actes qui pour se faire reconnaître, exigent la prise en compte de leur contexte d'énonciation. Cette conception conduit à dépasser la fragmentation typologique qui oppose figures de mots et figures de pensée. Le langage figuré met en scène une énonciation problématisée qui invite à réfléchir sur une forme de modalisation. L'analyse couplée de deux figures connues du moins-disant, l'euphémisme et la litote, tente d'expliquer la divergence pragmatique qu'on leur prête dans beaucoup de traités (faire entendre moins vs " réveiller l'idée du plus »). Si une confrontation de points vue sous-tend ici l'acte d'atténuation, le point de vue autre qui traverse alors l'énonciation puise à deux sources différentes: un dialogisme interdiscursif pour l'euphémisme, un dialogisme interlocutif pour la litote.

Bertrand Verine traite des hyperboles vives, comprises comme énoncés intensifs sincères, dans le genre de la conversation ordinaire. Constatant que les approches référentielles et pragmatiques aboutissent à une problématique de l'exagération qui laisse entière la question de son échelle normative d'évaluation, on s'efforce de rendre compte de telles séquences en réintroduisant le locuteur-énonciateur dans sa double relation dialogique au destinataire et à l'interdiscours. On observe en effet que les marqueurs linguistiques d'intensité (prosodiques, lexicaux, morphologiques et/ou syntaxiques) cohabitent le plus souvent avec des marqueurs (plus ou moins explicites) de dialogisme permettant d'articuler au moins deux points de vue : le PDV normatif prêté à la doxa, à un tiers ou à l'interlocuteur, et le PDV hyperbolisant que le locuteur vise à faire adopter au destinataire. 